\title{
Calvarial Reconstruction of Small-Size Skull Defects Performing "8 Cranioplasty" in Children: Surgical Experience, Clinical Results and Treatment Algorithm
}

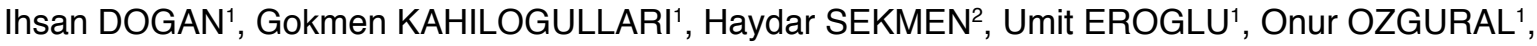 \\ Mustafa Agahan UNLU1 \\ ${ }^{1}$ Ankara University, School of Medicine, Department of Neurosurgery, Ankara, Turkey \\ ${ }^{2}$ Derince Education and Research Hospital, Department of Neurosurgery, Kocaeli, Turkey \\ This article was presented as an oral presentation $25^{\text {th }}$ Annual Congress of Turkish Neurosurgical Society, April 22-26, 2011, Antalya, Turkey.
}

\section{ABSTRACT}

AIM: To report our clinical experience, surgical treatment algorithm and technique in the reconstruction of uncomplicated and small-size calvarial defects by performing autologous split-bone grafting technique on anatomical findings and patients' outcome.

MATERIAL and METHODS: We covered the defective calvarial zone without bone (area, 3.8 to 7.5 centimetersquare; median area 4.2 centimetersquare) following the resection of pathological skull region with an inner table of horizontally split adjacent skull site in thirty-six patients (aged between 28 and 125 months; median age, 68.75 months, minimum follow-up 14 months). The donor site, the characteristics of skin incision such as length, shape, location and bone splitting technique were determined based on our treatment algorithm for " 8 cranioplasty". Pre- and postoperative clinical outcomes and courses as well as radiological results are documented.

RESULTS: Mean follow-up period for all children was 25 months. There was no mortality and 5.55\% morbidity ( $\mathrm{n}=2$ : 1 sterile wound dehiscence and 1 sterile wound discharge). No infected flap was seen and none of the patients required a second surgery. No graft failure, tumor recurrence or residue occurred.

CONCLUSION: Almost all of our patients reported good surgical and clinical outcomes with this presented management and surgical algorithm. Autologous split bone grafting technique in small-size skull defects, with its proposed name "8 cranioplasty" is a safe and effective reconstructive procedure due to its short-term surgery, small-size surgical zone, low complication rate, good cosmetic results and cost effectiveness. In addition, our algorithm specified for this procedure is purposive and also saves the surgeon time in the surgical planning stage.

KEYWORDS: Calvarium, Cranioplasty, Children, Defect

\section{INTRODUCTION}

$\mathrm{R}$ econstruction of the calvarial bone defects by placing various implant materials either autograft or allograft has been a commonly practiced surgical procedure since ancient civilizations $(2,18)$. Although the purposes of this procedure are cosmetic and functional recovery such as reconstitution of physiological barriers in all cases, the surgical technique and materials used in cranioplasty procedures vary depending on the etiology, concomitant diseases, size and location of the defect and age of the patient $(6,11,15)$. Good 
surgical results and patient outcome could only be achieved by the selection and use of appropriate material after taking into account these large number of variables under correctly applied surgical technique.

Physical strength, constructional stability and rigidity in the defective area, and the presence of tissue reaction due to biocompatibility of the chosen material are the other significant factors in selecting the appropriate graft type $(4,14,17)$. In addition to all these factors, autografts are preferred in the selection of graft in pediatric patients to prevent potential deformity due to the incomplete and continuing skull development (13).

This study describes a well-known cranioplasty technique involving our experience with various indications for small size and uncomplicated cranial defects in the pediatric age group at Ankara University, Ibn-i Sina Hospital. While this technique is widely performed and well known for the reconstruction of skull defects of different sizes depending on many pathologies and named as "autologous split-calvarial bone graft technique" according to its appearance after reconstruction of small size defects, we proposed to name it as "8 cranioplasty" in our article. Furthermore, taking into account scalp retraction, size of surgical working area and cosmetic concerns, we also explained a new preoperative surgical algorithm determining the shape and location of appropriate skin incision considering the cranioplasty region and the etiology, from which side of the calvarial defect cranitomy the bone graft should be performed and the horizontal splitting technique. We have designed our algorithm on these basic issues and also each surgical step is focused on and explained in detail. In the light of our experience, combining with data obtained from radiological imaging findings and intraoperative observations, we created the final state of surgical principles of the eight cranioplasty technique.

\section{- MATERIAL and METHODS}

\section{Patients and Diagnosis}

We reviewed the hospital records of patients who underwent cranioplasty with autologous split calvarium bone graft at the Ankara University, Ibn-i Sina Hospital between 2005 and 2013. We assessed 36 patients (22 boys, 14 girls) aged between 28 and 125 months (mean 68.75 months). Of the 36 calvarial defects, 15 were located in the frontal bone, 12 were in the parietal bone, 8 were in the occipital bone and 1 was in the temporal bone.

Patients' complaints are in a wide range from a palpable and growing mass and localized pain to softness, edema and depression feeling on the skull. X-ray is usually sufficient for pre-diagnosis (Figure 1A). Radiolucency of the pathologic zone may give hints about the lesion. Computed tomography (CT) findings are more valuable in terms of the presence of destruction of skull, expansion and invasion features of the lesion (Figures 1B, C). Although CT sections or axial (Figure 1D), sagittal and coronal magnetic resonance imaging (MRI) are helpful and effective in the diagnosis of the lesion, the threedimensional view of the skull following the reconstruction of these images provides an additional surgical perspective and contribution for planning the procedure (Figure 1E). In some cases where it is not possible to show the lesion on CT images especially in the parenchyma window (Figure 1C) due to its insufficiency, MRI is also preferable and more advantageous to demonstrate the pathology or in other situations whether the brain parenchyma is affected or not.

The defective calvarial zone without bone in consequence of surgically resected pathology or the pathology itself (fracture) was measured between 3.8 to 7.5 centimetersquare (median area 4.2 centimetersquare) by using free computer software (OsiriX Medical Imaging Software) on CT images (Figure 2). Autologous bone graft was obtained from horizontallysplit calvarial bone adjacent to the skull defect. All of our cranioplasty procedures, comprising the resection of the pathology until secure bone tissue was seen and coverage of the calvarial defect with autologous split bone graft, were performed in a single session. Follow-up periods of these patients were a minimum 14 months and at the end of the second-year the patients' follow-ups were ended. In this period of time, all the patients were evaluated in terms of wound healing, cosmetic results, strength and rigidity of grafts. Strength of the graft was used for describing the solidity and resistance of the split bones and the rigidity synonym for stability was used for describing the mobility of the bones in their settled surgical area. These two terms express constructional integrity and were evaluated radiographically and by palpation during physical examination. In the followup periods of our patients, we kept our physical examination findings in the forefront according to the radiological images. Due to the radiation exposure, we did not prefer to follow all of our patients radiologically. Parents' satisfaction of patients' condition was our criteria for determining the cosmetic results. Complications were recorded and evaluated as intraoperative, post-operative early period (first week after surgery), and postoperative late period (later than 1 week after surgery). We also pointed out our ways of coping with these complications. The bone pathologies deemed necessary for cranioplasty after total resection are shown in Table I.

\section{Preoperative Management, Surgical Decision and Surgical Technique}

Exposure of the cranial defect is the first surgical step as usual. It should be noted that skull region used as autograft has to be included in surgical working region. For this purpose, before the surgery, determination of certain localizations and size of skull pathology together with the adjacent healthy skull region chosen for cranioplasty is essential. From which side of the defect the graft material could be procured, the shape of the skin incision and the flap type could not be standardized for all settlements of calvarial defects. All of these surgical steps should be performed according to the pathology and specifically its settlement (Figures 3A, B). Thus, appropriate skin incision type and length have been chosen pursuant to our algorithm in all of our patients. According to the basic principles of our algorithm, we prefer to replace the optimal skin incision just behind the hairline for frontal bone localized small size lesions or defects specific to the forehead (Figure $3 \mathrm{C)}$. 

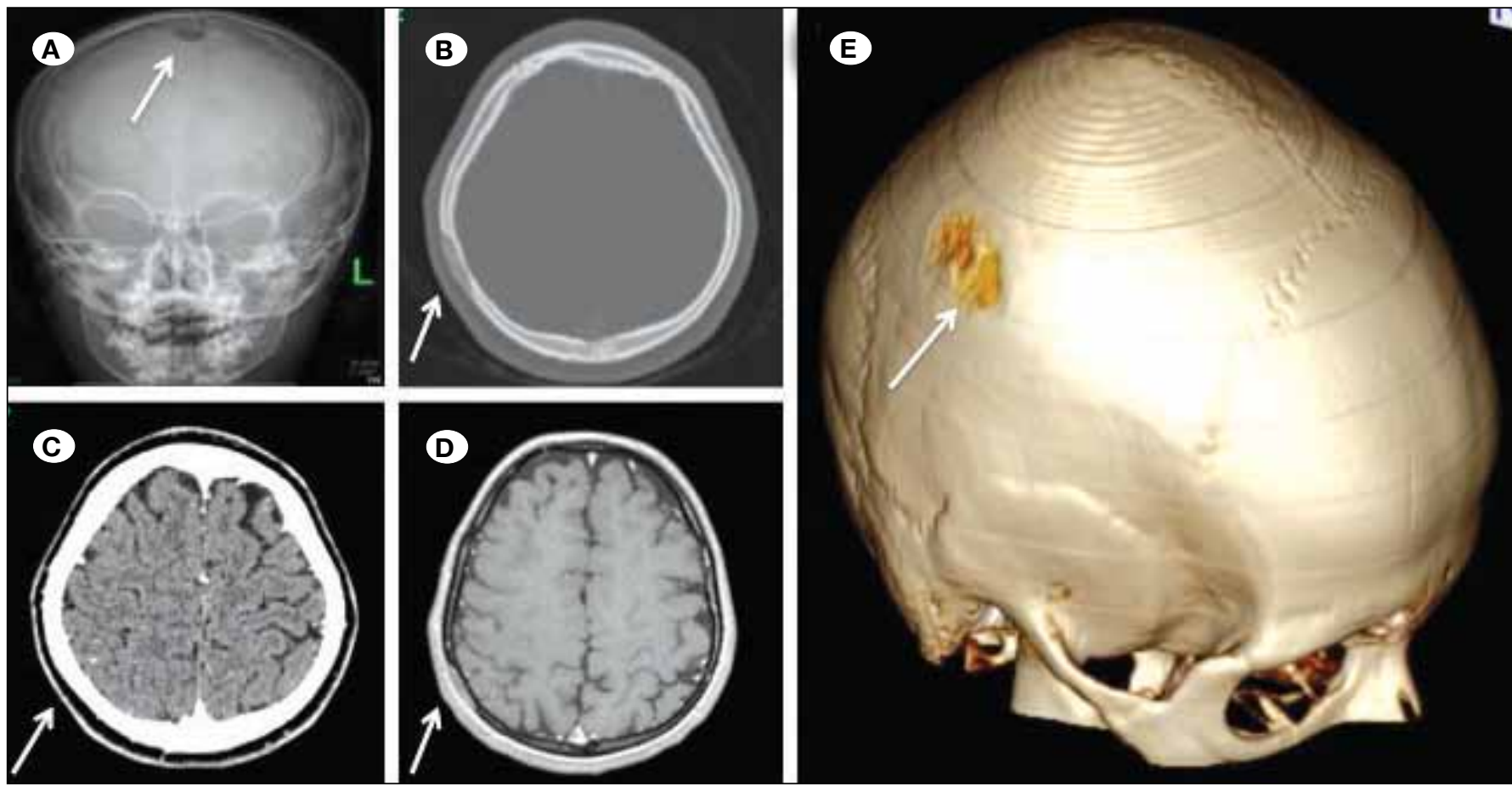

Figure 1: Radiological assessment and views of calvarial pathology of the same patient A) Plain X-ray cranium roentgenogram of a child patient showing a destructive radiopaque lesion at the midline. B, C) Bone window (B) and parenchyma window (C) CT imaging of the same patient. While the destruction of calvarial region due to the pathology is seen as a hypointense region on bone window, the calvarium seems intact in the same localization in the parenchyma window. D) Contrast enhancement T2 MRI of same pathology is showing a space-occupying intradiploic skull lesion E) 3-dimensional reconstruction of the cranium showing a lytic lesion on parietal bone. (On all images, an arrowhead shows the lesion).

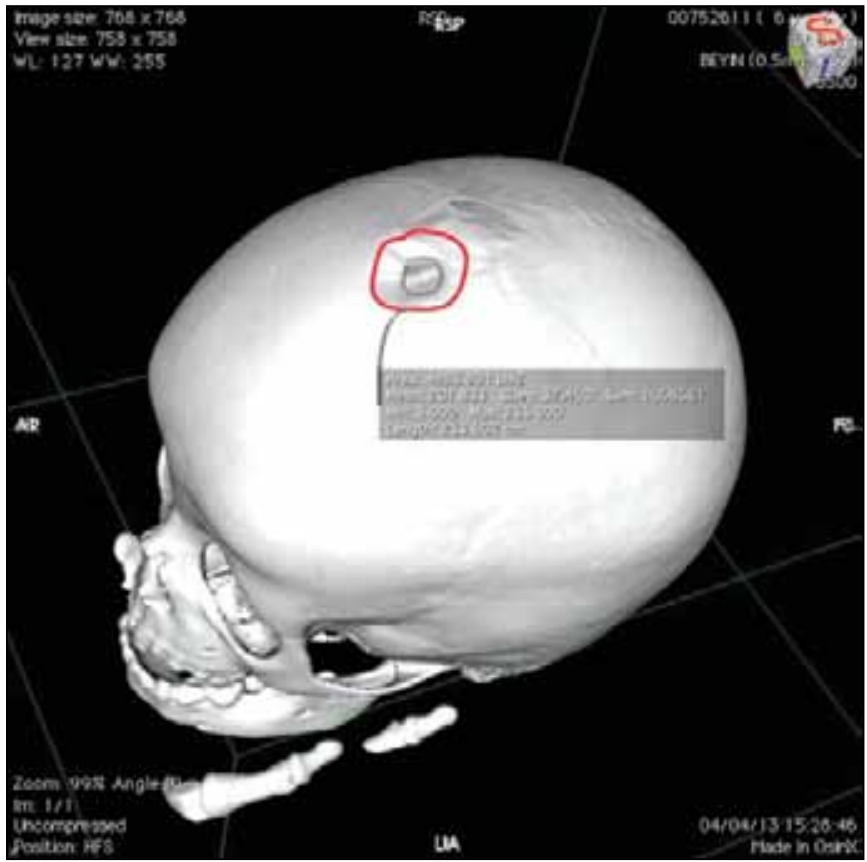

Figure 2: Measurement and demonstration of a destructive midline calvarial lesion in the 3-dimensional image of a child patient reconstructed by free computer software (OsiriX Medical Imaging Software) (Red line is showing the periphery of the lesion).
Table I: Indications for "8 Cranioplasty"

\begin{tabular}{lc}
\hline Indications & $\begin{array}{c}\text { Number of } \\
\text { patients }\end{array}$ \\
\hline Infection & 3 \\
\hline Trauma & 7 \\
\hline Intradiploic Dermoid Cyst & 6 \\
\hline Fibrous Dysplasia & 3 \\
\hline Eosinophilic Granuloma & 4 \\
\hline Growing Skull Fracture & 6 \\
\hline Craniectomy for Intracerebral Haematoma & 1 \\
\hline Intradiploic Epidermoid Cyst & 6 \\
\hline
\end{tabular}

For "8 cranioplasty" procedures, a linear skin incision is preferred generally in almost all cases except anterior frontal bone lesions as discussed before. The linear incision should be on the line that connects the centers of the calvarial pathologic zone and adjacent area (Figure 3D). This incision will provide adequate exposure for this procedure.

Subsequent to optimal exposure of the surgical field and total resection of the osseous lesion until solid and healthy bone tissue with smooth edges has been observed (Figure 4A), 
creating a pattern of the calvarial defect to be repaired is the next surgical step (Figure 4B). For this purpose, the defective empty region is filled with semi-solid formable stuff such as bone-wax. After boundaries of the model become clear, it is relocated to the solid skull region over the calvarium where cranitomy will be performed and the margin of this model is marked above the skull to create a similar pattern (Figure 4C). After craniotomy following the marked borders, an "8" shape calvarial cavity is created (Figure 4D).

The choice of craniotomy side to obtain the autograft is crucial. We refer to this region as the "craniotomy side" in our article. There is no standard decision for this event. It varies according to the settlement and the size of the defect. Vascularization of the skin flap, compatibility of thickness and concavity of the calvarial bone graft for splitting and replacing are major determining factors in the choice of craniotomy side. For this purpose, and to define the anatomic site of the calvarial defect to simplify our surgical plan and make our algorithm understandable; we separated frontal, parietal and occipital bones into three surgical regions and these regions were also divided into three subregions (Table II and Figure $5 A-C)$. In the cause of reconstruction of the calvarial defects of each settlement, craniotomy sides were determined and shown separately and specifically (Figures 5A-C). For temporal bone defects, we recommend to obtain autograft from the parietal bone. Parietal bone could be thought as a good option depending on its thickness. Temporal bone is not a good alternative source for autografts because of its thin structure. Incision over the temporal muscle and disruption of vascularization may eventually cause muscular atrophy and thus, poor cosmetic results may occur.

Splitting of autologous calvarial bone (Figure $6 \mathrm{~A}$ ) is performed with an oscillating saw (Figures 6B; 7A, B). This device can be thought as one of the best or only option for achieving two similar bone pieces in almost every cases (Figure 6C) that will be used for "8 cranioplasty" (Figure 6D). Splitting of small size calvarial grafts of pediatric age group patients needs more attention and experience than adults. Due to the thin intertabular space, grafts can be broken into several pieces, have irregular shapes and thickness during this procedure. Asymmetric twin flaps will cause poor cosmetic results. Therefore, splitting of the craniotomised bone either with oscillating saw (Figure 7A, B) or No. 20 blade (Figures $7 \mathrm{C}-\mathrm{F}$ ) should follow the direction from the edge to the center (Figure $7 E)$. As a recently defined surgical practice, using a No. 20 blade may be dangerous for the surgeon. To avoid potential hazards, both the handle of the blade and the calvarial graft must properly be in contact with firm ground (Figure 7D).

The final surgical step is the reconstruction of the 8-shaped defective zone (Figure 8A), after removing the pathological zone and craniotomising an adjacent intact bone with similar

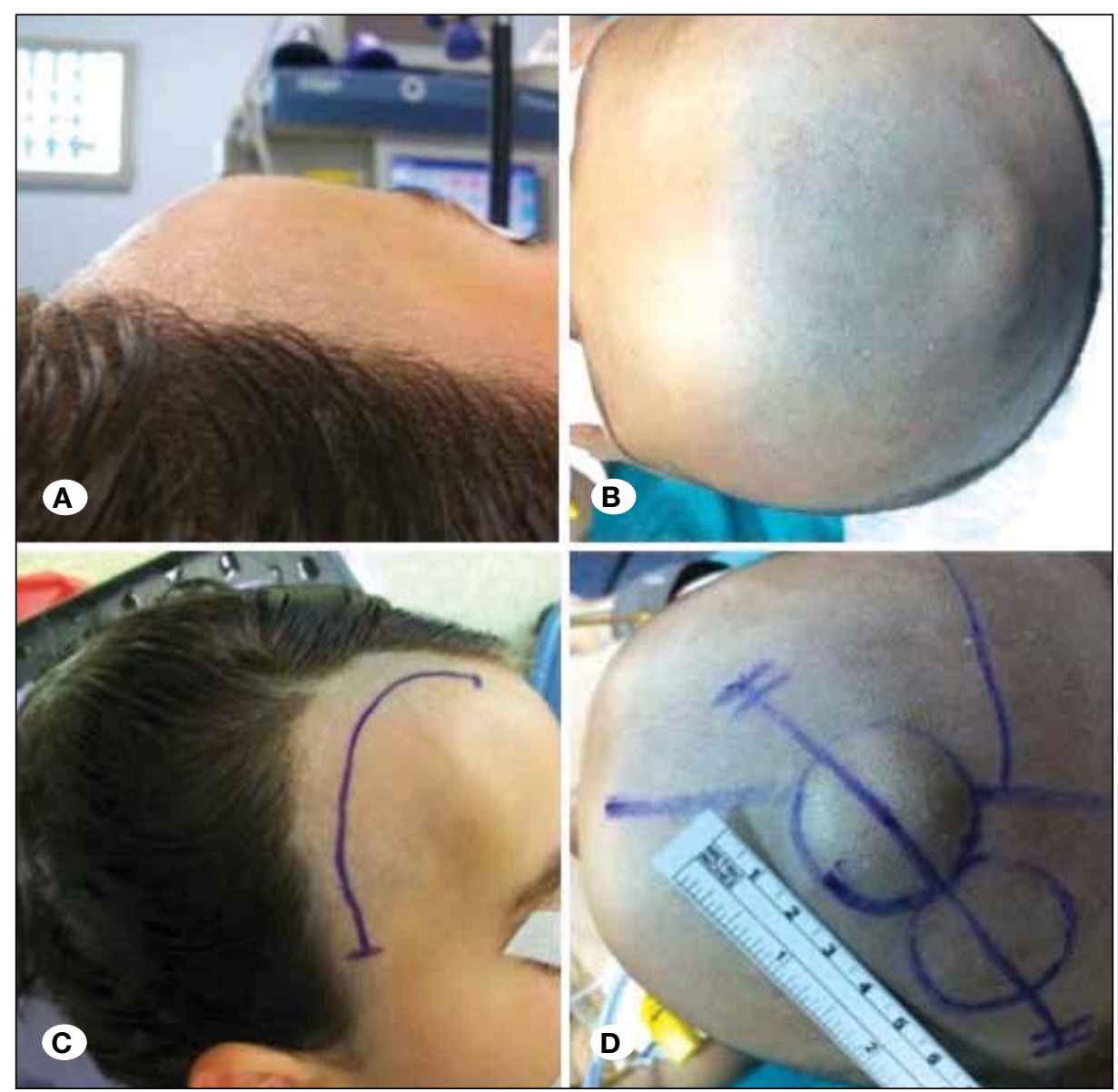

Figure 3: A, B) Palpable mass below the scalp leads to a cosmetic disorder in the frontal region of the skull (A) and on the midline just in front of the coronal suture (B). C) Surgical patient position for frontal bone localized lesion and skin incision is designed in a curvilinear fashion just behind the hairline. Elasticity and mobility of scalp enable accessing the pathologic region although the skin incision is away from lesion. D) Skin incision that cross the centers of both the pathologic area itself and its craniotomy side extending toward both directions to create a large surgical field prepared for the midline pathology. 

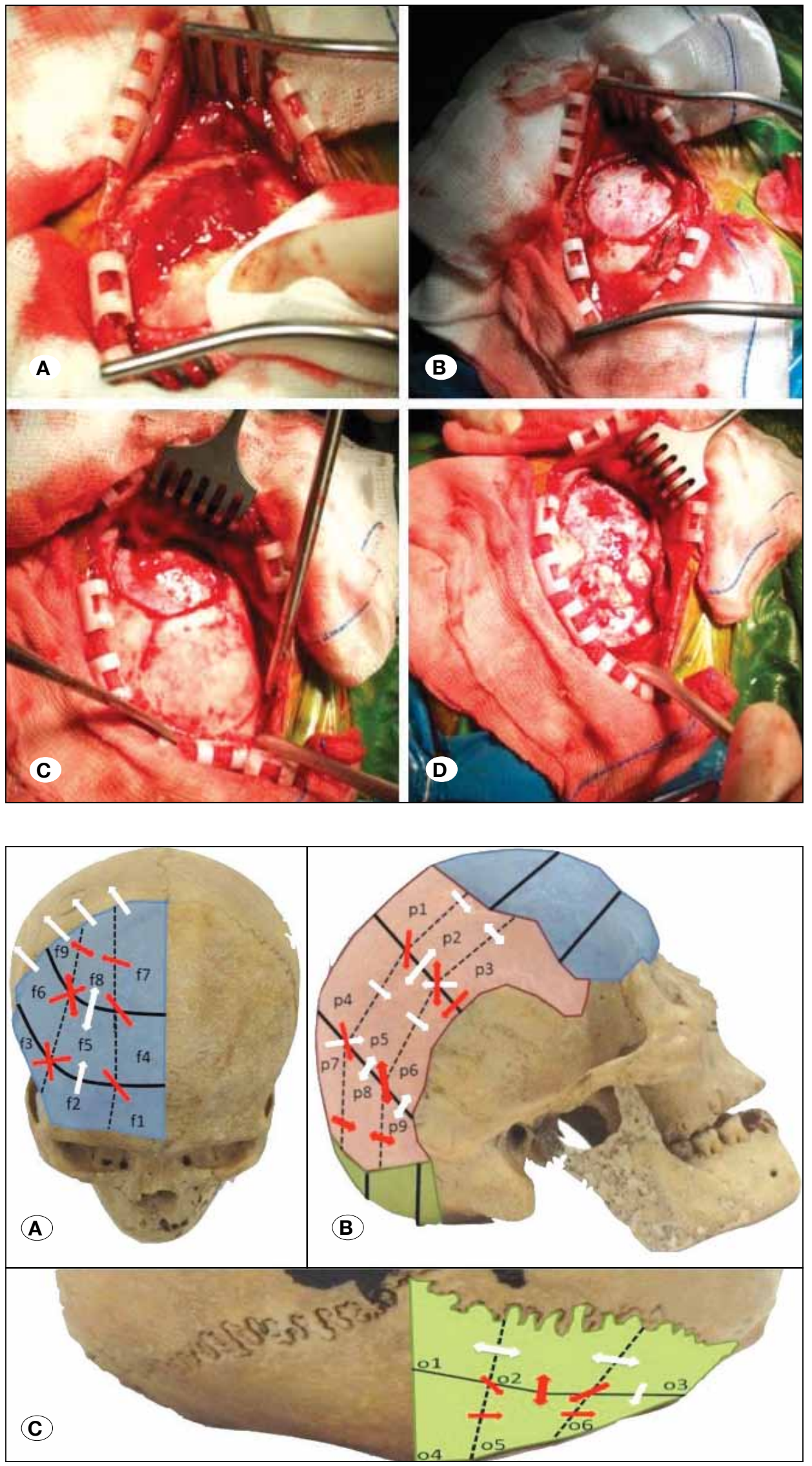

Figure 4: A) Exposure of osseous lesion with nearby solid calvarial region from where autologous graft will be used. B) Totally resected calvarial bone site needs to be repaired and the surgical working region enlarged owing to the elasticity of scalp. C) Pattern of the defective region is created in the craniotomy side with the same size and shape over the surface of the skull. D) After craniotomy of the healthy and solid calvarial bone used for the autograft, two fields symmetrical to each other imitating number " 8 " are created.
Figure 5: Surgical regions of frontal bone (A), parietal bone (B), occipital bone (C) and craniotomized side of calvarial defect according to their settlements (red arrows show the primarily chosen side of grafting region and white ones show the secondary regions). 
pattern of this zone that will be used as a graft material together. Inner and outer tables of intact bone graft are connected to each other and placed into the 8-shaped defective zone to maintain the calvarial integrity and surface smoothness
(Figure 8B). It is recommended to cover the pathologic region with inner table of the calvarial graft while the outer table stays in its original position.

Table II: Surgical Regions of Skull Bones for Defining the Anatomic Site of the Calvarial Defect Adapted to "8 Cranioplasty" (Inferior Region of Occipital Bone Below the Nuchal Line not Included)

\begin{tabular}{cccc}
\hline Skull Bone & & Regions-Subregions & \\
\hline \multirow{3}{*}{ Frontal } & Anterior 1/3 & Middle 1/3 & Posterior 1/3 \\
& Lateral (f3) & Lateral (f6) & Cateral (f9) \\
& Center (f2) & Center (f5) & Medial (f7) \\
& Medial (f1) & Medial(f4) & Posterior 1/3 \\
& Anterior 1/3 & Middle 1/3 & Lateral (p9) \\
& Lateral (p3) & Lateral (p6) & Center (p8) \\
Parietal & Center (p2) & Center (p5) & Medial (p7) \\
& Medial (p1) & Medial (p4) & Posterior 1/2 \\
& Anterior 1/2 & & Lateral (o6) \\
& Lateral (o3) & & Center (o5) \\
& Center (o2) & & Medial (o4) \\
\hline
\end{tabular}
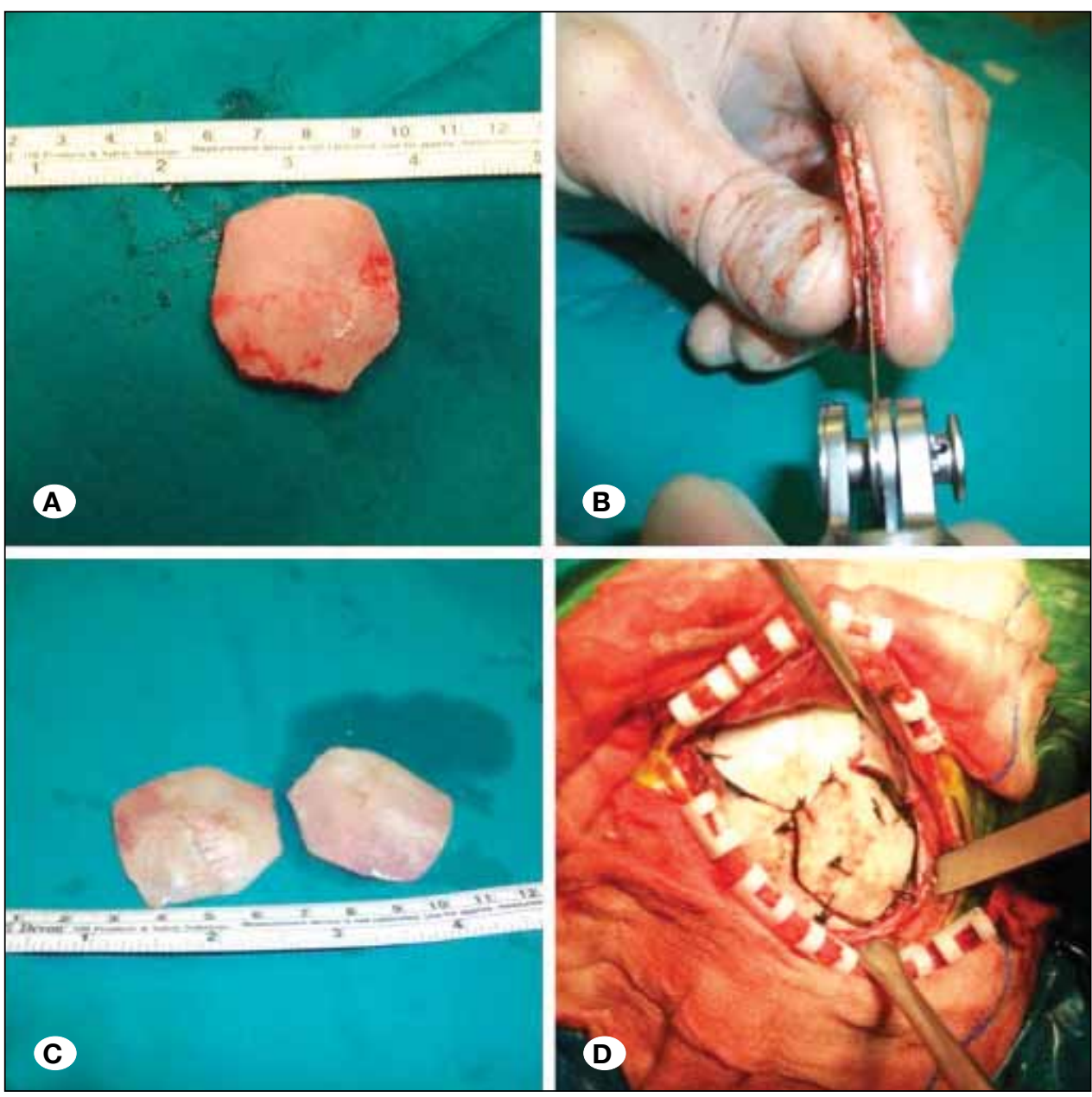

Figure 6: A) Craniotomized bone flap. B) Horizontal splitting of craniotomized bone flap into inner and outer tables with the oscillating saw. C) Inner and outer tables of craniotomized bone flap identical to each other will be used as two separate graft materials. D) Reconstruction of the defective calvarial zone by placing and fastening up the grafts. 


\section{RESULTS}

Each patient was evaluated during surgery, the postoperative early period (first week after surgery) and the late post-operative period (later than 1 week after surgery). The postoperative follow-up period ranged from 16 to 49 months. Mean length of hospital stay of our patients was 28 hours (16 hours to 145 hours). No mortality and no bone resorption occurred in our patients. A second surgical procedure was not required for any patient. During surgery, the bone graft got divided into two pieces vertically throughout the suture line after craniotomy in one patient. These two pieces split separately and then inner tables and outer tables were connected among themselves. Afterward, the procedure continued in its standard way. In one of the patients, sterile wound discharge occurred in the early postoperative period (3 days later surgery) and daily wound care with local drainage was performed and the discharge gradually decreased without any medical treatment. One patient developed a skin flap infection 48 hours after the surgery and was treated in
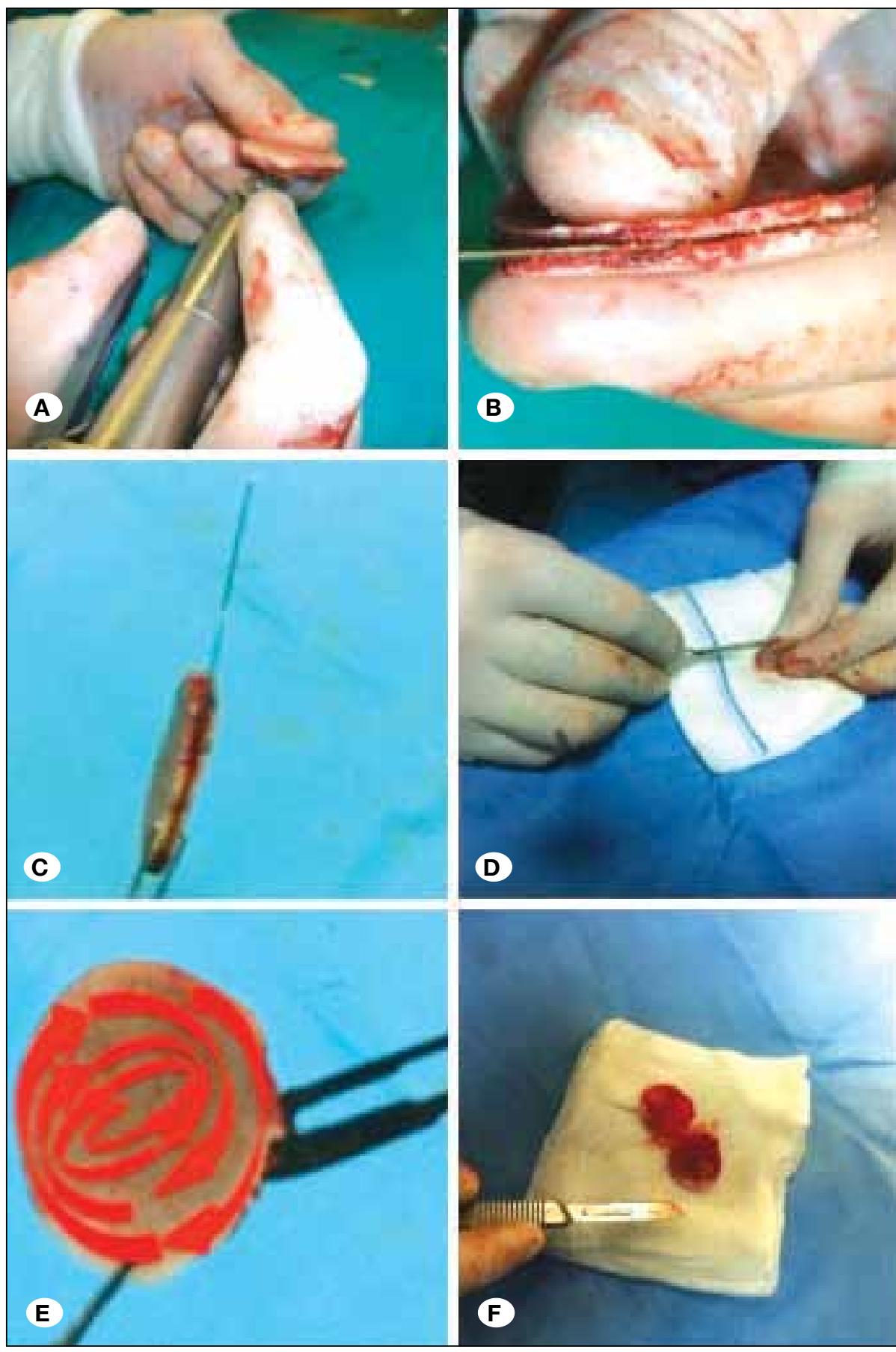

Figure 7: A, B) Splitting of craniotomized bone graft by ossilating saw and close view of inner and outer tables, intertabular space. C-E) Another proposed alternative splitting technique using a no. 20 blade especially for younger pediatric patients and thinner calvarial grafts. Circular movement of blade from the periphery to the center (E) will provide good results in terms of obtaining two similar grafts (F) (Red curved arrows indicate the splitting direction of craniotomized bone graft sequentially). 
about 2 weeks with systemic antibiotic therapy. In the late postoperative period, no recurrent infection of this patient developed and no primary infection was observed in any other patient. Cosmetic and constructional results were evaluated 6 months after surgery. In all patients who had undergone radiological imaging, radiographically bone density of the grafts had almost the same radioopacity with the nearby calvarial bone (Figures 9 A-D). The border between the grafts and adjacent bone tissue with which the grafts combined disappeared in all of the patients. Except for 4 patients, all the grafts were rigid in their settled place 6 months after the operation. In postoperative $12^{\text {th }}$ month, the rest of the grafts
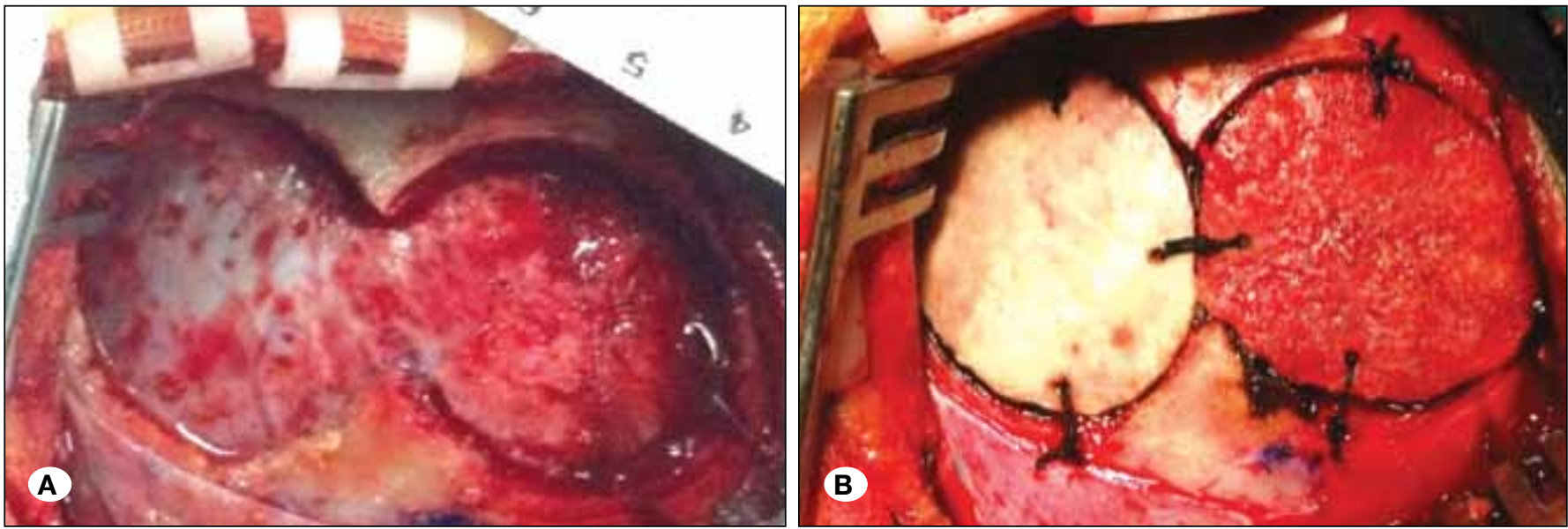

Figure 8: A) 8-shaped skull defect after removing both calvarial lesion and graft. B) 8-shaped reconstruction with inner (right) and outer (left) tables of intact craniotomized graft.
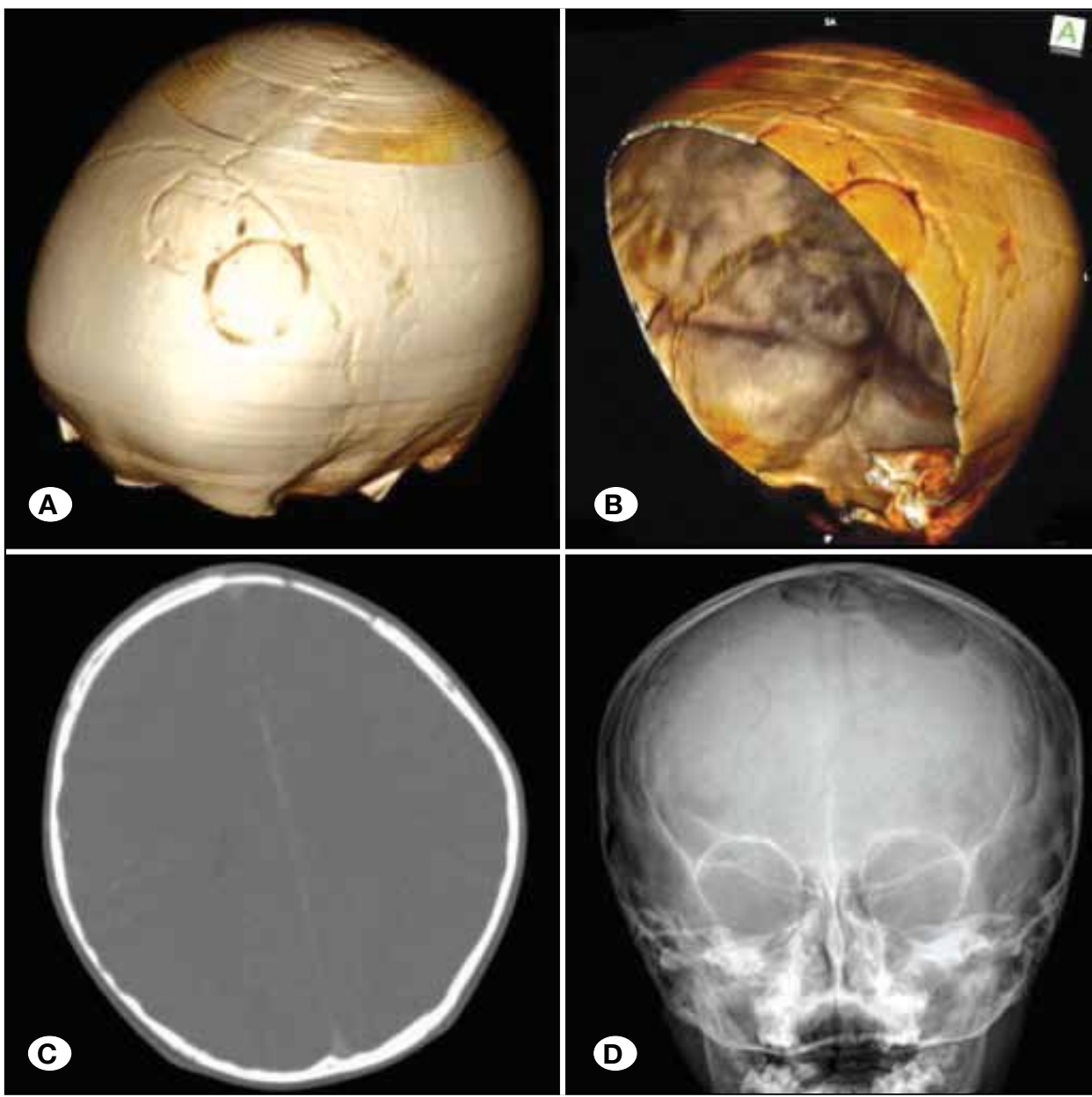

Figure 9: Postoperative radiological images after "8 cranioplasty". A, B) 3-dimensional views after procedure demonstrating the contour fitting and fusion of the grafts (A) and calvarial surface smoothness (B). C) Axial CT image is demonstrating the continuity in calvarial integrity and inclination. D) Plain X-ray roentgenogram after reconstruction. 
Table III: Scaling Criteria for Determining the Graft's Condition and Appearance of the Incision Site and Surgical Skin Area

\begin{tabular}{l}
\hline Physical examination and inspection findings \\
\hline With palpation, no calvarial graft mobility. No scalp deformity on inspection \\
\hline With palpation, minimal calvarial irregularity but no scalp deformity \\
\hline Minimal depression/elevation in operation field when looking closer, no calvarial graft mobility +/- scalp deformity \\
$\begin{array}{l}\text { suggesting construct failure or insufficiency } \\
\text { Evident depression/elevation in operation field when looking from a distance, presence of calvarial graft mobility }+/-\end{array}$ \\
$\begin{array}{l}\text { scalp deformity suggesting construct failure or insufficiency } \\
\text { Presence of scar tissue }+/-, \text { an open wound }+/- \text { depression or elevation on scalp }\end{array}$ \\
\hline
\end{tabular}

became stable. Patient satisfaction were assessed by their rating over five points with a mean satisfaction score of 4.36 (5 patients 3 points, 13 patients 4 points and 18 patients 5 points). In the determination of the patients' or parents' satisfaction, when scoring, they were asked to take into account the patients' adaptation to social life after surgery, occurrence and duration of postoperative symptoms and their cosmetic acclaim. Also we generated an objective scale and scoring system that should be assessed by physicians for the purpose of the determination of both the graft rigidity and cosmetic results together (Table III). According to our scale, 3 out of 36 patients got 3 points, 6 out of 36 got 4 points and 27 out of 36 got 5 points at the end of the first year. No scalp deformity or calvarial graft mobility was found in our patients.

\section{DISCUSSION}

In the selection of appropriate material for the purpose of restoring the calvarial defect, the age of the patient is the most decisive criteria. In situations where the calvarial bone adjacent to defective zone is intact and the patient is in the pediatric age group, an autologous bone graft should be the first option $(9,14)$.

Previously craniectomised bone flaps, split calvarium bone or rib grafts are autologous cranioplasty alternatives for repairing calvarial defects (19). For the purpose of repairing calvarial defects in children due to continuing skull enlargement in this patient age group, in the choice of graft material, predominance of autologous bone grafts compared to allograft ones is due to their biologic coherency and morphologic conformity.

In the absence of a suitable craniectomised flap or bank calvarial bone, or when the shape of the original defect has changed drastically, the best option is the utilisation of split calvarium or rib grafts $(8,9,12-14,20)$. The term "8 cranioplasty" is used in our article for describing the calvarial defects repaired by upturned or directly placed inner table of the adjacent splitted bone horizontally. "8 cranioplasty" could be accepted as subtype of autologous split bone graft technique performed for small size defects. Although "8 cranioplasty" is a feasible and practicable technique, it also has disadvantages for repairing extensive and large cranial defects. Constructional similarity between the defective calvarial site and donor site is impaired in proportion to the widening size of pathology. Concavity, curvature, thickness, neighborhood with superficial vascular structures such as sagittal, transverse sinuses of donor calvarial bone are major determinant features about whether "8 cranioplasty" will be performed or not. In the event that one of these features is incompatible, the place where the symmetry of the defective site in the other side of the skull is chosen as donor site or other type of graft materials could be used. When there is an intact calvarial bone tissue between sites where cranioplasty has been performed, we do not use the term " 8 cranioplasty".

Three-dimensional CT images and multi-axial 2 dimensional CT images were also valuable and useful for the purpose of choosing the proper graft side of the calvarial defect during the preoperative preparation period. Principally, on radiological examination, it should be considered that the curvature and concavity of the graft should be compatible with the defective zone. According to our study and the newly determined algorithm, each region and compatibility of these regions with each other in their specific locations in terms of their curving pattern were noted and examined radiologically. When the concavity or curving pattern of each cranial bone (frontal (Figure 10B, C), parietal (Figure 10B-E), occipital (Figure 10 B-E)) were analyzed separately from the others radiologically, it could be realized that almost any region of cranium is compatible with the surrounding bone in its own cranial bone limits. For providing the optimum alignment and natural appearance after grafting postoperatively, we simplified and generalized the grafting sides as 2, 5, 7 and 10 o'clock sides of defective zone except for the exceptions. In general, we proposed the grafting sides from a line that cut the cranium that is angled 45 degrees with the horizontal plane anteriorly (Figure $10 \mathrm{~A}-\mathrm{E})$.

In situations where larger cranial defects have to be repaired by autologous split calvarial bone, the preferred donor site of the calvarium is usually the opposite site of the defect symmetrically. The shape of the craniotomised site or harvest area is approximately the mirror image of the region that requires cranioplasty where the sagittal suture is chosen as the midline between these two regions (1). Splitthickness cranial bone grafts can also be used to replace numerous smaller fractures fragments in addition to calvarial pathologies. Despite all these advantages, a longer skin incision, especially bicoronal incision, and larger operative 
field can be distinguished as disadvantages of this technique. For these reasons, the donor site of the region that needs to be restored should be chosen from the adjacent calvarial region if possible. Considering the size and location of the defect; "8 cranioplasty" should be preferred as the first surgical option. On the other hand, alternative techniques may be regarded instead of " 8 cranioplasty" in defects that do not allow covering by surrounding split bone structure. Preoperative radiological evaluation of the defect for determining the surgical strategy may also be helpful.

Unlike other neurosurgical procedures for any pathology, cosmetic results are important as much as clinical outcomes for "8 cranioplasty". Most of the patients usually have cosmetic complaints and almost all of them have no neurologic deficit. For these reasons, as the initial step of this procedure, the skin incision type, length, location are determinant features of the cosmetic outcome. Thus, the care in making the skin incision should be the same as removing the pathology and repairing the defective zone.

As we have demonstrated in our study, calvarial defects may not be seen exactly in regions that we illustrated nor should they be of the same size with these regions (Figures $5 \mathrm{~A}-\mathrm{C}$ ). Our aim here is to show that each one of these three bones has a slope peculiar to itself by creating a main principle in the surgeon's mind, and taking into account the curvature or curving pattern of cranial bones for the purpose of choosing the proper graft region. Regardless of the location of the defect, we believe our illustration provides a guideline in the determination of the region for graft material.

No studies discussing the calvarial bone grafting technique have reported any complication about resorption and loss of calvarial transplants $(3,5,7,10,16)$. Thus, fresh autologous bone is considered as the best suitable material for reconstruction of cranial defects in view of its anatomofunctional fusion of the graft with the adjacent bone as well as the possibility of partial or total revitalization of the graft itself $(1,2)$.

In our technique, we determined the adjacent craniotomised site of the calvarial defect on a imaginary line that intersects the horizontal axis at an angle of 135 degrees. According to this, both the center of the defect and the donor site should be on this line. In contrast, a line that intersects the horizontal axis at an angle of 45 degrees angle could be another option. Each calvarial bone has its own concavity and when these bones, i.e. the frontal, parietal and occipital bones, are divided into 3 or 2 equal subregions, it is practically indicated that each subregion could be the donor side of the others in same bone.

As we described in the details in our technique, a linear skin incision is preferred in almost every case. In some cases, according to the pathology and etiology, and especially in patients with traumatic head injury, skin defects may be observed (Figures 11 A-D). These conditions may be in a very wide range from full-thickness incisions to abrasions. According to the neighborhood between the settlement of the calvarial defect and traumatic skin incision, it is recommended that the surgical incision should cover the previously formed skin defect for cosmetic results, wound healing and arterial supply of the skin flap. In patients with traumatic head injury and skin laceration (Figure 11A), the surgical skin incision should include the skin laceration for good cosmetic results and vascularization of skin flap (Figure 11B), and the shape of skin incision may differ based on the size and location of the defective region.
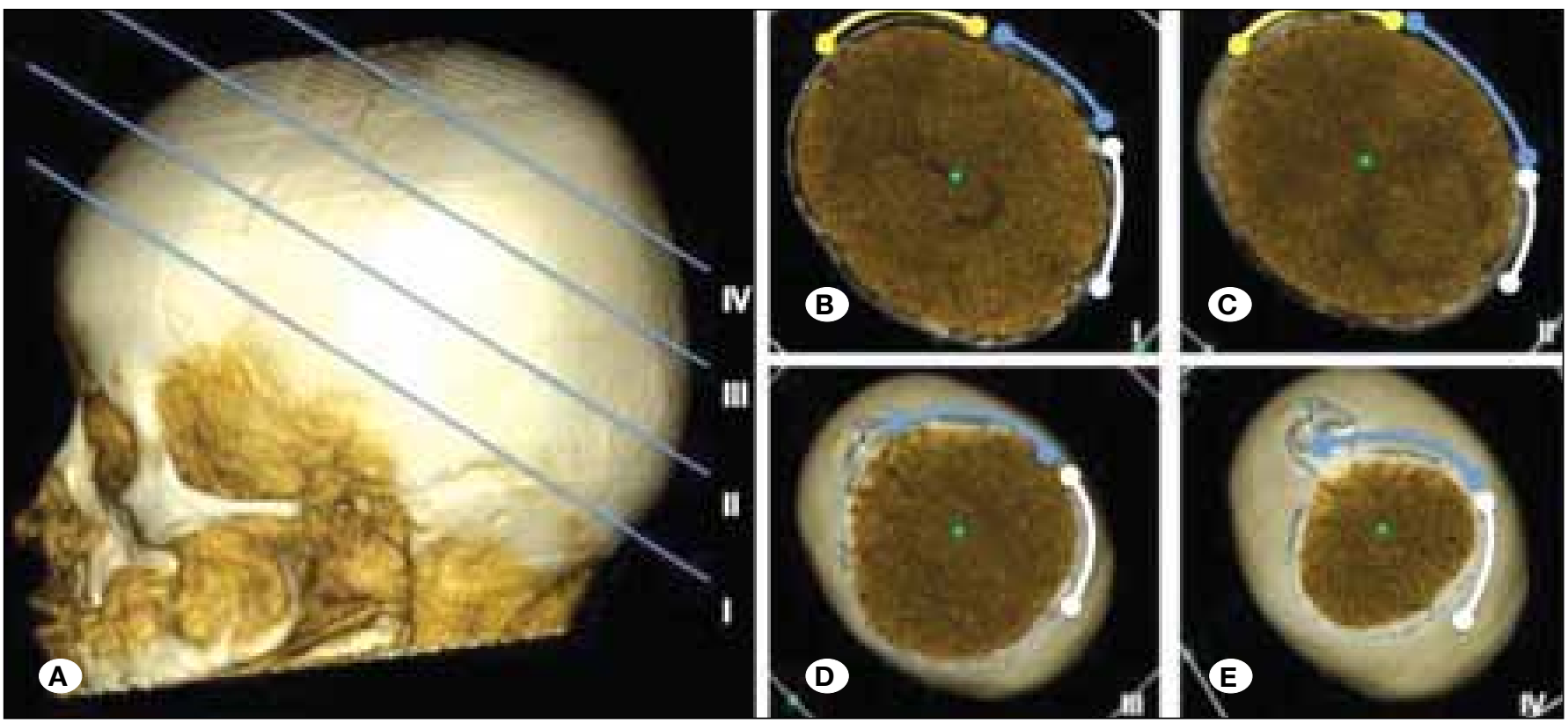

Figure 10: Axial images of slices view from above cutting horizontal plane with 45 degrees of angle (A) starting from cranial base to vertex respectively (B-E). (B, C, D, E on the right side showing the axial view of the skull sections I, II, III, IV from superior respectively) (Yellow, blue and white curved lines are showing the convexity and curvature of frontal, parietal and occipital bones respectively). 
Additionally, wound infection, cerebrospinal fluid (CSF) fistula and leptomeningeal cyst occurred in a patient who was excluded from our study because "8 cranioplasty" was not performed. Due to the size of a defective region caused by an epidermoid cyst that was almost $2.5 \mathrm{~cm}^{2}$, reconstruction was not performed. Here in this case, it may be concluded that the any calvarial defect independent of its size should be repaired.

In children, the scalp is more flexible and mobile than adults. Neurosurgeon can take advantage of these features to minimalize the skin incision and skin flap for good cosmetic results. Due to the excess of elastic connective tissue and loose connection of periosteum with bone and skin, this situation provides a large surgical working area with short skin exposure.

Not only the clinical importance of the autologous implants biological adaptation to defective calvarial zone also the reshaping and integration with the enlarging skull assigns them the material of choice for skull reconstruction. In children, incorporation of the bone flap is thought to occur by a combination of osteoconduction and osteoinduction. Regardless of the type of autologous material, surgical attention should be focused on protecting the integrity and configuration of the dura, periosteum and bone flap because of their high potential of inducing the organic matrix of the bone graft to transform its pluripotential osteoprogenitory cells into osteoblasts and chondroblasts. Although in split-bone technique, bone integrity is discomposed artificially, splitting the calvarial graft into two symmetric segments consisting of similar layers is essential. As we demonstrated in our patients' follow-up radiological assessments, having all the histological layer of the grafts despite corrupted integrity resulted in high affinity with adjacent bone (Figure 12).

Contour fitting of split autologous bone graft is below constructional expectations during surgery and early postoperative period. Losing bone tissue from the circumference of the bone graft during craniotomy prevents fitting of bone graft to the defective zone completely. Initially, this situation gives rise to worse osseo-integration. As a result of insufficient osteoconduction, the stability of the autologous bone graft could not be at the desired level. In pediatric patients, due to enhanced ability to integrate with the growing skull, contours of the grafts both among themselves and the solid bone tissue gradually approach each other and maintain the calvarial bone integrity in post operative late periods $(9,14)$.
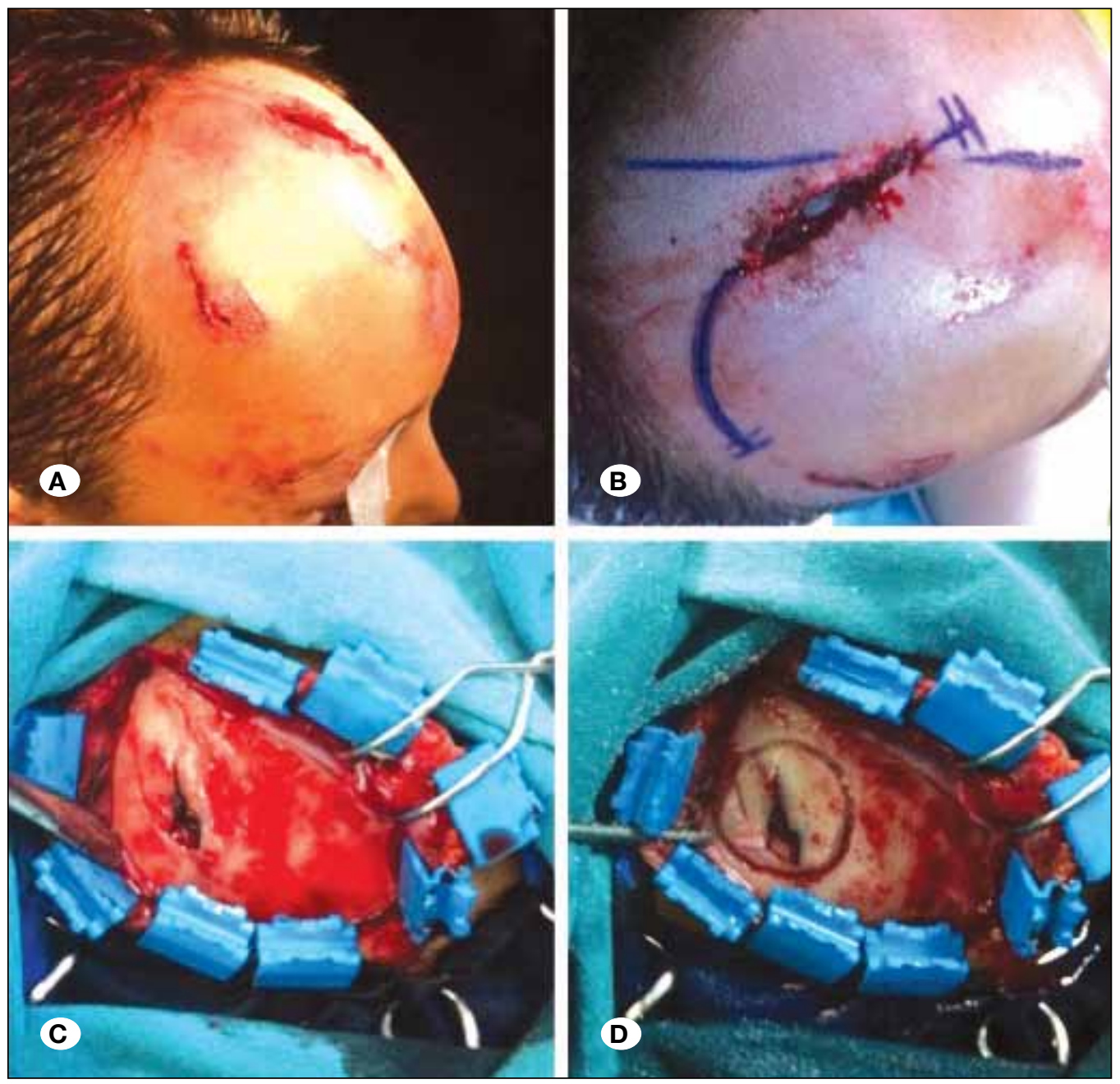

Figure 11: Traumatic scalp incision (A) and surgical skin incision (B) adjusted to previously occured skin laceration. In patients with depressed fracture (C), previously occurred traumatic scalp cut should be carried out to view the surgical 8-shaped field (D). 


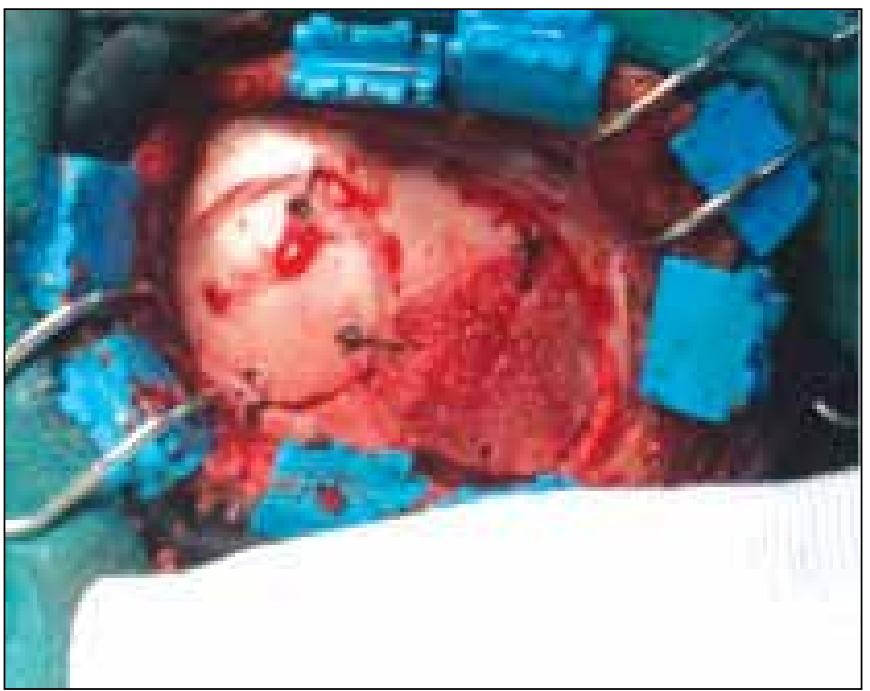

Figure 12: "8 cranioplasty".

Inoue et al. pointed out that cranioplasty with split-thickness calvarial bone is not possible in younger patients (11). In their report, the patients were between the ages 7 and 9 . Due to the under developed diploe and insufficient thickness, they did not split the calvarium in patients under 7 years old. In contrast to this argument, we performed our technique in much younger patients (28 months). Correct and proper usage of no 10 blade in younger patients would allow splitting thinner calvarium. When splitting the graft in this method, the wrists of the surgeon and bone graft should be on table to avoid possible injury as discussed previously (Figure 7D).

In addition, according to advances in technology, electronic devices such as bone-cusas could find a place in splitting the calvaria of younger patients. In contrast to our splitting technique, use of these devices is expensive and increases the total duration of this procedure.

\section{- CONCLUSION}

"8 cranioplasty" should be considered as the first surgical option for the reconstruction of small size and non-complicated calvarial defects. In addition to being an effective, relatively inexpensive and short-term procedure, it is also a safe surgical technique with low complication and no mortality rate. Besides all of these advantages, the use of a blade for splitting the intact calvarial segment allows this cranioplasty technique to be implemented on much younger children. Eventually, our algorithm specifically created for this cranioplasty type, guides the neurosurgeon in surgical planning and is therefore practicable and purposive.

\section{- REFERENCES}

1. Agrawal A, Garg LN: Split calvarial bone graft for the reconstruction of skull defects. J Surg Tech Case Report 3: 13-16, 2011
2. Artico M, Ferrante L, Pastore FS, Ramundo EO, Cantarelli D, Scopelliti D: Bone autografting of the calvaria and craniofacial skeleton: Historical background, surgical results in a series of 15 patients, and review of the literature. Surg Neurol 60: 7179, 2003

3. Brusati R, Biglioli F, Mortini P, Raffaini M, Goisis M: Reconstruction of the orbital walls in surgery of the skull base for benign neoplasms. Int J Oral Maxillofac Surg 29: 325-330, 2000

4. Ducic Y: Titanium mesh and hydroxyapatite cement cranioplasty: A report of 20 cases. J Oral Maxillofacial Surg 60: 272-276, 2002

5. Frodel JL: Calvarial bone graft harvest in children. Otolaryngol Head Neck Surg 121: 78-81, 1999

6. Gil Z, Abergel A, Leider_Trejo L, Khafif A, Margalit N, Amir A, Gur E, Fliss DM: A comprehensive algorithm for anterior skull base reconstruction after oncological resections. Skull Base 17: 25-37, 2007

7. Gruss JS, Mackinson SE, Kassel EE, Cooper PW: The role of primary bone grafting in complex craniomaxillofacial trauma. Plast Reconstr Surg 75: 17-24, 1985

8. Gupta SK, Reddy NM, Khosla VK, Mathuriya SN, Shama BS, Pathak A, Tewari K, Kak VK: Growing skull fractures: A clinical study of 41 patients. Acta Neurochir (Wien) 139:928-932, 1997

9. Hockley AD, Goldin JH, Wake MJC, lqbal J: Skull repair in children. Pediatr Neurosurg 16:271-275, 1990-1991

10. Hunter D, Baker S, Sobol SM: Split calvarial grafts in maxillofacial reconstruction. Otolaryngol Head Neck Surg 102: 345-350, 1990

11. Inoue A, Satoh S, Sekiguchi K, Ibuchi $Y$, Katoh S, Ota K, Fujimori S: Cranioplasty with split-thickness calvarial bone. Neurol Med Chir (Tokyo) 35:804-807, 1995

12. Iwama T, Yamada J, Imai S, Shinoda J, Funakoshi T, Sakai $\mathrm{N}$ : The use of frozen autogenous bone flaps in delayed cranioplasty revisited. Neurosurgery 52: 591-596, 2003

13. Josan VA, Sgouros S, Walsh AR, Dover MS, Nishikawa H, Hockley AD: Cranioplasty in children. Childs Nerv Syst 21: 200-204, 2005

14. Moreira-Gonzalez A, Jackson IT, Miyawaki T, Barakat K, DiNick V: Clinical outcome in cranioplasty: Critical review in long-term follow-up. J Craniofac Surg 14:144-153, 2003

15. Neligan PC, Boyd JB: Reconstruction of the cranial base defect. Clin Plast Surg 22: 71-77, 1995

16. Powell NB, Riley RW: Facial contouring with outer-table calvarial bone: A 4 year experience. Arch Otolaryngol Head Neck Surg 115: 1454-1458, 1989

17. Rish BL, Dilon JD, Meirowsky AM, Caveness WF, Mohr JP, Kistler PJ, Weiss GH: Cranioplasty: A review of 1030 cases of penetrating head injury. Neurosurgery 4: 381-385, 1979

18. Sanan A, Haines SJ: Repairing holes in the head: A history of cranioplasty. Neurosurgery 40: 588-603, 1997

19. Taggard DA, Menezes AH: Successful use of rib grafts for cranioplasty in children. Pediatr Neurosurg 34: 149-155, 2001

20. Weber RS, Kearns DB, Smith RJ: Split calvarium cranioplasty. Arch Otolaryngol Head Neck Surg 113: 84, 1987 